\title{
Mixed Problem for Hyperbolic Systems of First Order
}

\author{
By \\ Masaru TANIGUCHI $*$ )
}

\section{Introduction}

In the last ten years, a general theory of mixed problems for linear hyperbolic systems of first order has been developed.

In this paper, we treat the $L^{2}$ well-posed mixed problems for strongly hyperbolic systems of first order with constant coefficients. It is well known that Cauchy problem for hyperbolic systems of first order with constant coefficients is $L^{2}$ well-posed if and only if it is strongly hyperbolic [12]. The family of strongly hyperbolic systems contains the strictly and symmetric hyperbolic systems. But, Strang's condition for strong hyperbolicity seems to be useless except for Cauchy problem. So, in Sec. 3, we prove another condition for strong hyperbolicity which is useful for not only Cauchy problem, but also mixed problems, the lacunas of Riemann's matrix of strongly hyperbolic systems [1], the propagation of singularities [8] and others. The $L^{2}$ well-posed mixed problems for the strictly and symmetric hyperbolic systems have been fully investigated. But, even if hyperbolic systems have constant coefficients, nothing is known in general for ones with multiple characteristics except for systems with constant multiple characteristics [10] and $2 \times 2$ systems [13].

We consider the mixed problem for hyperbolic systems of first order with constant coefficients:

Received June 29, 1972.

Communicated by S. Matsuura.

*) Department of Mathematics, Science University of Tokyo, Tokyo, Japan. 


$$
\left\{\begin{array}{l}
\frac{\partial u}{\partial t}=A \frac{\partial u}{\partial x}+B \frac{\partial u}{\partial y}+f(t, x, y)=L[u]+f \\
u(0, x, y)=0 \\
S \cdot u(t, 0, y)=g(t, y)
\end{array}\right.
$$

in a quarter space $\left.\{t, x, y) \mid t \geqq 0, x \geqq 0, y \in R^{1}\right\}$, where $u$ is an $N$-vector, $A$ and $B$ are $N \times N$ real constant matrices, and $S$ is an $l \times N$ constant matrix with rank $l$. Already, L. Sarason [11], T. Sadamatsu [10], H. O. Kreiss [7] and K. Kajitani [5] gave a priori estimates for hyperbolic systems. But they did not treat the system with multiple characteristics except for T. Sadamatsu. Here, we treat the problem (1.1) with multiple characteristics. In Sec. 4, we prove some lemmas for the problem (1.1) which are similar to Kreiss's paper. In Sec. 5, we treat the problems occurring by reason of multiple characteristics with the help of the result in Sec. 3, and prove the Main Theorem. The author expresses his thanks to Professor H. Sunouchi for many useful advices.

\section{Assumptions and Result}

We consider the mixed problem

$$
\left\{\begin{array}{l}
\frac{\partial u}{\partial t}=A \frac{\partial u}{\partial x}+B \frac{\partial u}{\partial y}+f(t, x, y)=L[u]+f \\
u(0, x, y)=0 \\
S \cdot u(t, 0, y)=g(t, y)
\end{array}\right.
$$

in a quarter space $\left\{(t, x, y) \mid t \geqq 0, x \geqq 0, y \in R^{1}\right\}$.

We assume the following conditions for (1.1):

Condition $\mathbb{I}$. The operator $\left(\frac{\partial}{\partial t}-L\right)$ is strongly hyperbolic, that is, for any $(\xi, \eta) \in R^{2}-\{0\}, A \xi+B \eta$ has only real eigenvalues and is uniformly diagonalizable in $(\xi, \eta)$.

Condition II. $A$ is non-singular and has the form 


$$
\begin{aligned}
& A=\left(\begin{array}{cc}
A_{I} & 0 \\
0 & A_{I I}
\end{array}\right)
\end{aligned}
$$

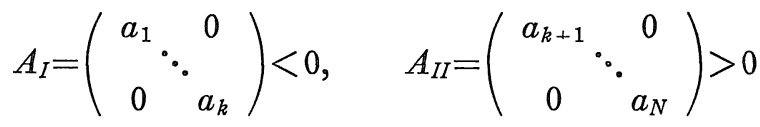

which is no restriction.

Condition III. The real roots of the equation $\operatorname{det}(\gamma I-(A \xi+B \eta))$ $=0$ with respect to $\xi$ are at most double for any $(\gamma, \eta) \in R^{2}-\{0\}$.

Put $M(\tau, \eta)=A^{-1}(\tau I-i \eta B)$, Re $\tau>0, \eta \in R^{1}$. We denote by $E_{-}(\tau, \eta)$ the span of the ordinary and generalized eigenvectors of $M(\tau, \eta)$ corresponding to the eigenvalues with negative real parts. The hyperbolicity of $\left(\frac{\partial}{\partial t}-L\right)$ implies that $\operatorname{dim} E_{-}(\tau, \eta)$ is constant for $\operatorname{Re} \tau>0, \eta \in R^{1}$ and equal to the number of negative eigenvalues of $A$ (cf. Lemma 4.1). We can locally construct a system of vectors $\left\{\boldsymbol{r}_{j}(\tau, \eta)\right\}_{j=1, \ldots, k}$ which is a base of $E_{-}(\tau, \eta)(\operatorname{Re} \tau>0)$, and the vectors $\boldsymbol{r}_{1}(\tau, \eta), \ldots, \boldsymbol{r}_{k}(\tau, \eta)$ are linearly independent, continuous and homogeneous of degree 0 in $\tau$ and $\eta(\operatorname{Re} \tau \geqq$ 0 ). This fact follows from the results of Sec. 4, Sec. 5 and Kreiss's paper.

Condition $\mathbb{I V}$. The boundary matrix $S$ satisfies the uniform Lopatinski's condition, that is, $l=k$ and $\operatorname{det}(S \cdot H(\tau, \eta)) \neq 0$ for $\operatorname{Re} \tau \geqq 0$, $\eta \in R^{1},(\tau, \eta) \neq 0$, where $H(\tau, \eta)=\left(r_{1}(\tau, \eta), \ldots, \pi_{k}(\tau, \eta)\right)$.

Taking the Laplace transform of $u$ with respect to $t$ and the Fourier transform of $u$ with respect to $y$, we get the problem of a system of ordinary differential equations depending on parameters with an inhomogeneous boundary condition:

$$
\begin{cases}\frac{d \hat{u}}{d x}=A^{-1}(\tau I-i \eta B) \hat{u}-A^{-1} \hat{f}, & x \geqq 0 \\ S \hat{u}=\hat{g}, & x=0\end{cases}
$$

where $\hat{h}(\tau, x, \eta)$ denotes the Fourier-Laplace transform of $h(t, x, y)$.

We obtain the following:

Main Theorem. Assume the Conditions I, II, III and IV. Then there exists a positive number $\mu_{0}$ such that, for any solution $\hat{u}(\tau, x, \eta)$ 
of the problem (2.1),

$$
\begin{aligned}
& \mu\|\hat{u}(\tau, \cdot, \eta)\|_{L^{2}\left(R_{+}^{1}\right)}^{2}+|\hat{u}(\tau, 0, \eta)|^{2} \\
& \quad \leqq \text { const. }\left\{\frac{1}{\mu}\|\hat{f}(\tau, \cdot, \eta)\|_{L^{2}\left(R_{+}^{1}\right)}^{2}+|\hat{g}(\tau, \eta)|^{2}\right\}
\end{aligned}
$$

for any $\operatorname{Re} \tau=\mu \geqq \mu_{0}$, where the constant does not depend on $\tau$ and $\eta$.

\section{Strongly Hyperbolic Systems with Two Space Variables}

We sum up some results in perturbation theory for matrices by T. Kato [6].

Let $N \times N$ matrix $T(\kappa)$ be $T^{(1)}+\kappa T^{(2)}\left(\kappa \in C^{1}\right)$, and the eigenvalues and the associated eigenprojections of $\mathrm{T}(\kappa)$ be $\lambda_{h}(\kappa)$ and $P_{h}(\kappa)$. We have the following results by $T$. Kato:

(K.1) Let $\kappa=0$ is an exceptional point of $P_{h}(\kappa)$.

If $P_{h}(\kappa)$ is represented by a Laurent's series in $\kappa^{1 / p}$, its principal part is finite.

(K.2) If $\lambda_{h}(\kappa)$ is single valued near an exceptional point $\kappa=\kappa_{0}$, then $P_{h}(\kappa)$ is single valued there.

(K.3) If $\kappa=\kappa_{0}$ is a branch point of $\lambda_{h}(\kappa)$ of order $p \geqq 2$, then $P_{h}(\kappa)$ has a pole there and $\left\|P_{h}(\kappa)\right\| \rightarrow \infty$ for $\kappa \rightarrow \kappa_{0}$.

Now, we return to the problem for strong hyperbolicity. Throughout Sec. 3, we assume that $A$ and $B$ are complex matrices. G. Strang shows the following conditions are equivalent:

(S.1) Operator $\left(\frac{\partial}{\partial t}-A \frac{\partial}{\partial x}-B \frac{\partial}{\partial y}\right)$ is strongly hyperbolic, that is, for any $(\xi, \eta) \in R^{2}-\{0\}, C(\xi, \eta)=A \xi+B \eta$ has only real eigenvalues and is uniformly diagonalizable in $(\xi, \eta)$.

(S.2) For any $(\xi, \eta) \in R^{2}-\{0\}$, any $z \in \mathbb{C}^{1}(\operatorname{Re} z>0)$, $\| \operatorname{Re} z(z I-i(A \xi$ $+B \eta))^{-1} \| \leqq$ const.

Theorem 3.1. The following conditions are equivalent:

(i) Operator $\left(\frac{\partial}{\partial t}-A \frac{\partial}{\partial x}-B \frac{\partial}{\partial y}\right)$ is strongly hyperbolic. 
(ii) (a) For any $(\xi, \eta) \in R^{2}-\{0\}, C(\xi, \eta)=A \xi+B \eta$ has only real eigenvalues and is diagonalizable.

(b) For any $(\xi, \eta) \in R^{2}-\{0\}$, the eigenvalues and the associated eigenprojections of $C\left(w_{1}, w_{2}\right)=A w_{1}+B w_{2}$ are holomorphic in a neighborhood of $(\xi, \eta)$ in $\mathbb{C}^{2}$.

Proof. (ii) $\Rightarrow(\mathrm{i}) . \quad C(\xi, \eta)$ is homogeneous of degree 1 in $\xi$ and $\eta$, and (ii)-(b) holds. So, $C(\xi, \eta)$ is uniformly diagonalizable in $(\xi, \eta)$ for any $(\xi, \eta) \in R^{2}-\{0\}$. Therefore (i) holds.

(i) $\Rightarrow$ (ii). From the condition (i), (ii)-(a) follows easily. To prove the condition (ii)-(b), we use the equivalent condition (S.2) for strong hyperbolicity. As $C(\xi, \eta)$ is diagonalizable for any $(\xi, \eta) \in R^{2}-\{0\}, C(\xi, \eta)$ has the spectral decomposition:

$$
C(\xi, \eta)=\sum_{j} \lambda_{j}(\xi, \eta) P_{j}(\xi, \eta)
$$

where $P_{j}(\xi, \eta)$ are mutually orthogonal projections. Therefore,

$$
(z I-i C(\xi, \eta))^{-1}=\sum_{j} \frac{P_{j}(\xi, \eta)}{z-i \lambda_{j}(\xi, \eta)} \quad:{ }^{\forall}(\xi, \eta) \in R^{2}-\{0\} .
$$

Let $z=\varepsilon+i \lambda_{k}(\xi, \eta)(\varepsilon>0)$. Then, by (S.2)

$$
\left\|P_{j}(\xi, \eta)\right\|=\lim _{\varepsilon \rightarrow+0}\left\|\varepsilon \sum_{j} \frac{P_{j}}{\varepsilon+i\left(\lambda_{k}-\lambda_{j}\right)}\right\| \leqq \text { const. }
$$

Without loss of generality, we may assume $\xi \neq 0$. Set $\kappa=w_{1} / w_{2}$ and $C^{\prime}(\kappa)=A+\kappa B$. Then, the eigenvalues $\lambda_{i}^{\prime}(\kappa)$ and the associated eigenprojections $P_{i}^{\prime}(\kappa)$ of $C^{\prime}(\kappa)$ are holomorphic at $\kappa=\eta / \xi\left((\xi, \eta) \in R^{2}-\{0\}, \xi \neq 0\right)$. For, if $\kappa=\eta / \xi$ is a branch point of $\lambda_{i}^{\prime}(\kappa)$ of order of $p \geqq 2$, the inequality $\left\|P_{i}^{\prime}(\eta / \xi)\right\| \leqq$ const. contradicts (K.3). So, $\lambda_{i}^{\prime}(\kappa)$ is holomorphic at $\kappa=\eta / \xi$. By (K.2), $P_{i}^{\prime}(\kappa)$ is single valued near $\kappa=\eta / \xi$. Also, by (K.1) and (K.3), $P_{i}^{\prime}(\kappa)$ is not able to have a pole there. Therefore, the eigenvalues and the associated eigenprojections of $C\left(w_{1}, w_{2}\right)$ are holomorphic in a neighborhood of $(\xi, \eta)$ in $\mathbb{C}^{2}$ for any $(\xi, \eta) \in R^{2}-\{0\}$.

Q.E.D.

Remark 1. By Theorem 3.1, we can construct a smooth symmetrizer 
for $\left(A \frac{\partial}{\partial x}+B \frac{\partial}{\partial y}\right)$ under strong hyperbolicity $[2],[3]$.

\section{Some Lemmas}

In this section, we shall derive some lemmas for the problem (1.1) which are similar to Kreiss's paper.

Lemma 4. $\mathbb{1}$ (R. Hersh [4]). For $\operatorname{Re} \tau>0, M(\tau, \eta)=A^{-1}(\tau I-i \eta B)$ has $k$ eigenvalues $\lambda$ with $\operatorname{Re} \lambda<0$ and $N-k$ eigenvalues $\lambda$ with $\operatorname{Re} \lambda>0$.

Proof. By the hyperbolicity of $\left(\frac{\partial}{\partial t}-L\right), M(\tau, \eta)$ has no eigenvalues $\lambda=i \xi$ where $\xi$ is real. The eigenvalues of $M(\tau, \eta)$ are continuous functions of $\tau$ and $\eta$. Let $\tau=1$ and $\eta=0$. Then we have Lemma 4.1.

Q.E.D.

Lemma 4.2 For $\operatorname{Re} \tau>0$, there exists an analytic transformation $U(\tau, \eta)$ such that

$$
U^{-1} M U=\left(\begin{array}{cc}
M_{11} & M_{12} \\
0 & M_{22}
\end{array}\right)
$$

Here, $M_{11}$ is a $k \times k$ matrix with eigenvalues $\lambda$ having negative real parts, and $M_{22}$ is an $(N-k) \times(N-k)$ matrix with eigenvalues $\lambda$ having positive real parts.

Lemma 4.2 follows from Lemma 4.1.

Lemma 4.3 For any $\tau(\operatorname{Re} \tau \neq 0)$ and pure imaginary $i \xi$ ( $\xi:$ real), we have the estimate

$$
\left\|(M(\tau, \eta)-i \xi I)^{-1}\right\| \leqq \text { const./|Re } \tau \mid
$$

Lemma 4.3 follows from the condition (S.2).

Let $\tau=\mu+i \nu, \mu, \nu$ real, $\mu \geqq 0$. We set

$$
\zeta=(i \nu, \eta), \quad \eta \in R^{1}
$$




$$
\begin{aligned}
M(\tau, \eta) & =M(\zeta, \mu)=A^{-1}((\mu+i \nu) I-i \eta B) \\
& =M(\zeta, 0)+\mu A^{-1}
\end{aligned}
$$

Lemma 4.4 For any fixed $\zeta_{0}=\left(i \nu_{0}, \eta_{0}\right) \neq 0$ and $\mu=0$, there exists a transformation $T_{0}$ such that

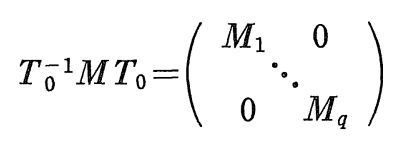

where $M_{j}$ have the following properties.

(i) The eigenvalues $\lambda$ of $M_{1}$ have $\operatorname{Re} \lambda \neq 0$.

(ii) The eigenvalues of $M_{j}(j \geqq 2)$ have $\operatorname{Re} \lambda=0$ and every $M_{j}(j \geqq 2)$ has one of the following three forms:

(1) $M_{j}\left(\zeta_{0}, 0\right)=\left(\lambda_{j}\left(\zeta_{0}, 0\right)\right)$

(2) $\quad M_{j}\left(\zeta_{0}, 0\right)=\left(\begin{array}{cc}\lambda_{j}\left(\zeta_{0}, 0\right) & i \\ 0 & \lambda_{j}\left(\zeta_{0}, 0\right)\end{array}\right)$

(3) $\quad M_{j}\left(\zeta_{0}, 0\right)=\left(\begin{array}{cc}\lambda_{j}\left(\zeta_{0}, 0\right) & 0 \\ 0 & \lambda_{j}\left(\zeta_{0}, 0\right)\end{array}\right)$

with $\lambda_{i} \neq \lambda_{j}(i \neq j)$.

Proof. Above forms are nothing else but a variant of Jordan's normal form. We have only the above forms by the Condition III.

Q.E.D.

Remark 2. The form (ii)-(3) occurs from the Condition I. We had not the form (ii)-(3) in Kreiss's paper. In Sec. 5, we treat the form (ii)(3).

\section{The Representation of $M(\zeta, \mu)$ by a Similar Transformation}

In Lemma 4.4, we have the form (ii)-(3) different from Kreiss's one. But we shall show that in such a case $M_{j}(\zeta, \mu)$ is diagonal in a neighbor- 
hood of $\left(\zeta_{0}, 0\right)\left(\zeta_{0} \neq 0\right)$.

Lemma 5.1 For any $\zeta_{0}=\left(i \nu_{0}, \eta_{0}\right) \neq 0, \mu=0$, there exists an analytic transformation $T(\zeta, \mu)$ of $(\zeta, \mu) \in U\left(\zeta_{0}, 0\right)$ where $U\left(\zeta_{0} 0\right)$ is a neighborhood of $\left(\zeta_{0}, 0\right)$, such that

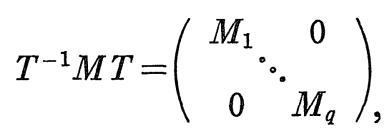

Furthermore, $M_{j}(\zeta, 0)(j \geqq 2)$ is pure imaginary, and if $M_{j}\left(\zeta_{0}, 0\right)(j \geqq 2)$ has the form

$$
M_{j}\left(\zeta_{0}, 0\right)=\left(\begin{array}{cc}
\lambda_{j}\left(\zeta_{0}, 0\right) & 0 \\
0 & \lambda_{j}\left(\zeta_{0}, 0\right)
\end{array}\right)
$$

$M_{j}(\zeta, \mu)$ has the form

$$
M_{j}(\zeta, \mu)=\left(\begin{array}{cc}
\lambda_{j 1}(\zeta, \mu) & 0 \\
0 & \lambda_{j 2}(\zeta, \mu)
\end{array}\right)
$$

Proof. When $M_{j}\left(\zeta_{0}, 0\right)$ has the form (i), or (ii)-(1), or (ii)-(2) in Lemma 4.4, we use Kreiss's paper to construct T. If $M_{j}\left(\zeta_{0}, 0\right)$ has the form (ii)-(3) in Lemma 4.4, the equation $\operatorname{det}\left(i \nu_{0} I-i\left(A \xi+B \eta_{0}\right)\right)=0$ with respect to $\xi$ has a double root $\xi_{0}=\lambda_{j}\left(\zeta_{0}, 0\right) / i$ by the Condition III. From the Condition I,

$$
\begin{gathered}
\operatorname{det}(\tau I-i(A \xi+B \eta))=\prod_{j=1}^{N}\left(\tau-i \gamma_{j}(\xi, \eta)\right) \\
:(\xi, \eta) \in R^{2}-\{0\}
\end{gathered}
$$

where $\gamma_{j}(\xi, \eta)$ is real $(1 \leqq j \leqq N)$. Without loss of generality, we may assume

$$
i \nu_{0}=i \gamma_{1}\left(\xi_{0}, \eta_{0}\right)=i \gamma_{2}\left(\xi_{0}, \eta_{0}\right)
$$

Then, by the Condition III and Theorem 3.1, 


$$
\frac{\partial}{\partial \xi} \gamma_{1}\left(\xi_{0}, \eta_{0}\right) \neq 0, \quad \frac{\partial}{\partial \xi} \gamma_{2}\left(\xi_{0}, \eta_{0}\right) \neq 0
$$

We consider the transformations

$$
\left\{\begin{array}{l}
w_{1}^{i}=\gamma_{i}(\xi, \eta) \\
w_{2}^{i}=\eta
\end{array} \quad(i=1,2) .\right.
$$

Then,

$$
\frac{\partial\left(w_{1}^{i}, w_{2}^{i}\right)}{\partial(\xi, \eta)}\left(\xi_{0}, \eta_{0}\right)=\frac{\partial}{\partial \xi} \gamma_{i}\left(\xi_{0}, \eta_{0}\right) \neq 0
$$

and $\gamma_{i}(\xi, \eta)$ is real for any $(\xi, \eta) \in R^{2}-\{0\},(i=1,2)$. So, by Theorem 3.1 and (5.4), the mappings (5.3) are topological and analytic mapping such that a neighborhood $U\left(\xi_{0}, \eta_{0}\right)$ of $\left(\xi_{0}, \eta_{0}\right)$ in $\mathbb{C}^{2}$ corresponds to a neighborhood $V\left(\nu_{0}, \eta_{0}\right)$ of $\left(\nu_{0}, \eta_{0}\right)$ in $\mathbb{C}^{2}$ and $U\left(\xi_{0}, \eta_{0}\right) \cap R^{2}$ corresponds to $V\left(\nu_{0}, \eta_{0}\right) \cap R^{2}$, where $\left(\xi_{0}, \eta_{0}\right) \in R^{2}-\{0\}$. Therefore, we can construct the analytic transformation $T$ in Lemma 5.1 .

We introduce the notation:

$$
|\zeta|^{2}=|\nu|^{2}+|\eta|^{2}, \quad \zeta^{\prime}=\zeta /|\zeta|, \quad \mu^{\prime}=\mu /|\zeta|
$$

and consider the matrix $M(\zeta, \mu)=|\zeta| M\left(\zeta^{\prime}, \mu^{\prime}\right)$ for a fixed $\zeta^{\prime}=\zeta_{0}^{\prime}$ as a function $|\zeta|, \mu^{\prime}$. Hereafter we consider in a neighborhood of $\left(\zeta_{0}, 0\right)$ $\left(\zeta_{0} \neq 0\right)$. By Lemma 5.1, every block $M_{j}(\zeta, \mu)$ has the form

$$
M_{j}(\zeta, \mu)=|\zeta|\left(M_{j}\left(\zeta_{0}^{\prime}\right)+\mu^{\prime} N_{j}\left(\zeta_{0}^{\prime}\right)+0\left(\mu^{\prime 2}\right)\right), N_{j}=\left(n_{i k}\right) .
$$

Lemma 5.2 (H. O. Kreiss [7] and J. V. Ralston [9]) Suppose that $s=s(j)$ is order of $M_{j}$. Then, there exists a constant $c>0$ such that the following hold in a neighborhood of $\zeta=\zeta_{0} \neq 0, \mu=0$ :

(i) If $M_{j}\left(\zeta_{0}, 0\right)$ has the form (ii)-(1) or (ii)-(2) in Lemma 4.4, $N_{j}\left(\zeta_{0}^{\prime}\right)$ is real and $\left|n_{s 1}\right|>c$.

(ii) If $M_{j}\left(\zeta_{0}, 0\right)$ has the form (ii)-(3) in Lemma 4.4, we have the inequalities $\left|\operatorname{Re} n_{11}\right|>c$ and $\left|\operatorname{Re} n_{22}\right|>c$.

Proof. See Kreiss's paper for (i). If $M_{j}\left(\zeta_{0}, 0\right)$ has the form (ii)-(3), 
we have

$$
M_{j}(\zeta, \mu)=|\zeta|\left(\begin{array}{cc}
\lambda_{j}\left(\zeta_{0}^{\prime}, 0\right)+\mu^{\prime} n_{11}+0\left(\mu^{\prime 2}\right) & 0 \\
0 & \lambda_{j}\left(\zeta_{0}^{\prime}, 0\right)+\mu^{\prime} n_{22}+0\left(\mu^{\prime 2}\right)
\end{array}\right) .
$$

So, by Lemma 4.3,

$$
\begin{aligned}
\frac{\text { const. }}{|\mu|} & \geqq\left\|T(M(\tau, \eta)-i \xi I)^{-1} T^{-1}\right\| \\
& \geqq\left|\frac{1}{\operatorname{Re} n_{i i^{\circ} \mu}+(\text { lower order of } \mu)}\right|
\end{aligned}
$$

where we set $i \xi=\left(\lambda_{j}\left(\zeta_{0}^{\prime}, 0\right)+\mu^{\prime}\left(i \operatorname{Im} n_{i i}\right)\right)|\zeta|(i=1,2)$. Therefore

$$
\left|\operatorname{Re} n_{11}\right|>c \text { and }\left|\operatorname{Re} n_{22}\right|>c . \quad \text { Q.E.D. }
$$

From Lemmas 4.1-4.4 and Lemmas 5.1-5.2, we can construct the symbol $R$ having the following properties by the method similar to Kreiss's paper:

(i) $R=R(\mu, \nu, \eta)$ is defined in $\left\{(\mu, \nu, \eta) \mid \mu \geq \mu_{0}>0,(\nu, \eta) \in R^{2}\right\}$ and a uniformly bounded smooth function of $\mu, \nu, \eta$ and of the coefficients of $A, B$ and $S$.

(ii) $R A$ is symmetric.

(iii) $w^{*} R A w \geqq \delta_{1}|w|^{2}-c|g|^{2}$ for all vectors $w$ which satisfy the boundary conditions.

(iv) $\operatorname{Re} R(\tau I-i \eta B) \geqq \delta_{2} \mu I_{9} \tau=\mu+i \nu$. Here $\delta_{1}, \delta_{2}>0$ and $c>0$ are constants independent of $\mu, \nu, \eta$.

Then, we get the Main Theorem.

\section{References}

[1] Bazer, J. and D.H.Y. Yen, Lacunas of the Riemann matrix of symmetric hyperbolic systems in two space variables, Comm. Pure Appl. Math. 22(1969), 279-333.

[2] Friedrichs, K. O., Pseudo Differential Operators, Lecture note in Courant Institute, 1967-68.

[3] Friedrichs, K. O. and P.D. Lax, Boundary value problems for first order operators, Comm. Pure Appl. Math. 18 (1965), 355-388. 
[4] Hersh, R., Mixed problems in several variables, J. Math. Mech. 12 (1963), 317334.

[5] Kajitani, K., First order hyperbolic mixed problems, J. Math. Kyoto Univ. 11 (1971), 449-484.

[6] Kato, T., Perturbation Theory for Linear Operators, Die Grundlehren der Math. Wiss., 132, Springer-Verlag, Berlin, 1966.

[7] Kreiss, H. O., Initial boundary value problems for hyperbolic systems, Comm. Pure Appl. Math. 23 (1970), 277-298.

[8] Ludwig, D. and B. Granoff, Propagation of singularities along characteristics with nonuniform multiplicity, J. Math. Anal. Appl. 21 (1968), 556-574.

[9] Ralston, J. V., Note on a paper of Kreiss, Comm. Pure Appl. Math. 24 (1971), 759-762.

[10] Sadamatsu, T., On mixed problems for first order hyperbolic systems with constant coefficients, J. Math. Kyoto Univ. 9 (1969), 339-361.

[11] Sarason, L., On hyperbolic mixed problems, Arch. Rational Mech. Anal. 18 (1965), 311-334.

[12] Strang, G., On strong hyperbolicity, J. Math. Kyoto Univ. 6 (1967), 397-417.

[13] - Hyperbolic initial boundary value problems in two unknowns, J. Differential Equations, 6 (1969), 161-171. 
